\section{Commentary on: Pre-operative radiographs for dental implants - are selection criteria being followed?}

\author{
S. J. J. McCrea
}

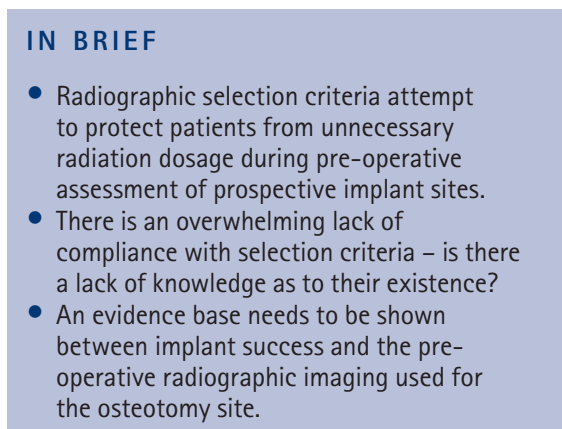

\begin{abstract}
Objectives To determine by anonymous survey whether the active membership of a particular dental society with variable training and clinical experience in dental implant insertion and restoration followed the published selection criteria for the pre-operative radiographic assessment of prospective dental implant osteotomy sites. Design Postal survey. Subjects and methods A postal questionnaire was sent to all professionally active members of the British Society of Periodontology $(n=560)$ during July 2005. The mailing was repeated in September 2005. Results Four hundred and fifty-nine questionnaires were returned a response rate of $81.5 \%$. One hundred and seventy-nine (39\%) respondents were not involved in placing or restoring dental implants and were excluded. Of the remaining 280 (61\%), 15 (5\%) clinicians limited themselves to placing implants, $85(30 \%)$ limited themselves to restoring implants, while $181(65 \%)$ both placed and restored their own implants. Two hundred and twenty-eight clinicians (80.8\%) did not follow UK selection criteria for single sites; 217 clinicians (77.5\%) did not follow the criteria for multiple sites. Two hundred and sixty-three clinicians (94\%) did not follow USA selection criteria. There were no statistically significant differences in compliance based on clinical experience or the number of implants placed. The only statistically significant difference was as a result of training those clinicians who had attended formal training courses were more likely to comply with published selection criteria. Significant differences also existed between the groups in the specific cross-sectional imaging modalities chosen. Conclusion 0ver 80\% of respondents are not following the UK or USA selection criteria for pre-implant imaging assessment. The results of this survey call into question the clinical relevance, usefulness, or knowledge of existing selection criteria. There is a need for new selection criteria developed by both radiologists and experienced implant clinicians.
\end{abstract}

\section{COMMENTARY}

Justification of radiographs is a hugely important topic. In all cases the benefit of the radiograph should outweigh the risk. In order to help with this justification process several groups have produced guidelines of when radiographs would be appropriate. ${ }^{1-4}$

This study looks specifically at the use of radiographs prior to implant placement. The postal questionnaire compares radiographic practice against published selection criteria from the Faculty of General Dental Practitioners (UK) and the American Academy of Oral and Maxillofacial Radiology (AAOMR)., The questionnaire was sent to the membership of the British Society of Periodontology. The response rate of $81.5 \%$ was excellent. The main findings were that over $80 \%$ of clinicians did not follow UK selection criteria for single implant use, 77.5\% did not follow UK selection criteria for multiple implant sites and that 94\% did not follow the selection criteria as described by the AAOMR.

The study highlights the variation in the recommendations between the two groups. What should be remembered at all times is that selection criteria are to be used as a guide. They should not be considered prescriptive, since each radiograph should be justified on an individual basis. For this reason there is always going to be some variation based on each individual's need. This variation may be exaggerated when the evidence to support the guidelines is small.

The paper states that the AAOMR recommend the use of cross-sectional imaging for all implant cases. However, clinical examination and conventional radiography will often be sufficient in simple cases, and following the AAOMR recommendations would lead to 'over imaging' of this patient group.

N. Drage, Dental Radiology Department, University Dental Hospital, Cardiff

1. Pendlebury M E, Horner K, Eaton K A (eds). Faculty of General Dental Practitioners (UK). Selection criteria for dental radiography. 2nd ed. London: Royal College of Surgeons of England, 2004.

2. Tyndall D, Brooks S (eds). American Academy of Oral and Maxillofacial Radiology. Selection criteria for dental implant site imaging: a position paper of the American Academy of Oral and Maxillofacial Radiology. Oral Surg Oral Med Oral Pathol Oral Radiol Endod 2000; 89: 630-637.

3. Harris D, Buser D, Dula K et al. E.A.O. guidelines for the use of diagnostic imaging in implant dentistry. A consensus workshop organized by the European Association for Osseointegration at Trinity College Dublin. Clin Oral Implants Res 2002; 13: 566-570.

4. European Commission. Radiation Protection 136. European guidelines on radiation protection in dental radiology. The safe use of radiographs in dental practice. EC, 2004.

DOI: 10.1038/sj.bdj.2008.526 